\title{
Rhabdomyolysis Associated Acute Kidney Injury Following a Physical Violence
}

\author{
Yalini Thivaharan $^{1^{*}}$ (D) and Indira Deepthi Gamage Kitulwatte ${ }^{2}$ \\ ${ }^{1}$ Postgraduate Trainee, Department of Forensic Medicine, Faculty of Medicine, University of Kelaniya, Sri Lanka \\ ${ }^{2}$ Professor, Department of Forensic Medicine, Faculty of Medicine, University of Kelaniya, Sri Lanka
}

*Corresponding author: Yalini Thivaharan, MD, Postgraduate Trainee in Forensic Medicine, Department of Forensic Medicine, Faculty of Medicine, University of Kelaniya, Sri Lanka, Tel: 0094777312349 , Fax: 0112959261

\begin{abstract}
Introduction: Physical violence can lead to serious and, rarely, fatal injuries. In addition to head injury, which is the leading cause of death and long-term disability, injuries of the musculoskeletal system and internal organs are important cause of assault-related morbidity. This paper discusses such rare complication of an interpersonal violence - rhabdomyolyis associated with Acute Kidney Injury (AKI).

Case: A 37-year-old man who claims to be assaulted by a group of people, presented with focal contusions and extensive grazed abrasions over the trunk and limbs. Injuries to brain and other visceral organs were excluded. Serum creatinine and urea were elevated significantly, along with increase in C-reactive protein and liver enzymes. Urinalysis contained red cells and leukocyte esterase, following which rhabdomyolysis was diagnosed. He developed oliguric AKI, and haemodialysis was initiated. He was discharged after eleven days of hospitalization, following improvement in renal functions.
\end{abstract}

Discussion: Rhabdomyolysis is a common cause for oliguric renal failure, and can be traumatic or non-traumatic. Rhabdomyolysis has specific clinical and laboratory parameters, but still requires high level of suspicion, for timely diagnosis. Highly elevated levels creatinine phosphokinase (CPK) is the most specific parameter for the diagnosis of rhabdomyolysis. Myoglobinuria, elevated levels of lactase dehydrogenase and transaminases are also considered valuable markers of rhabdomyolysis. AKI is the commonest systemic complication of rhabdomyolysis and various causative mechanisms have been explained.

Conclusion: Rhabdomyolysis requires high index of suspicion when acute kidney injury and altered metabolite levels are suspected in a patient with major or minor muscle injuries, in order to prevent complications or death.

\section{Keywords}

Rhabdomyolysis, Acute kidney injury, Creatinine phosphokinase, Muscle injuries

\section{Introduction}

Forensic pathologists, even though deal mainly with postmortem examinations, they also come across a wide variety of clinical cases, in their day-to-day practice. Patients with a history of physical violence/ assault predominate the number of cases seen per day. History of assault can vary from domestic violence to blunt and sharp force trauma inflicted upon one another during a brawl.

Physical violence can lead to serious and, rarely, fatal injuries. In addition to head injury, which is the leading cause of death and long-term disability, injuries of the musculoskeletal system and internal organs are important cause of assault-related morbidity [1]. Such instances can lead to immediate death or delayed death due to complications.

One should be mindful of the rare complications of trauma acquired by a victim, as high level of suspicion and expertise is required to diagnose such conditions. Some of such life-threatening conditions are handlebar hernias [2], traumatic abdominal wall hernias and evisceration [3], fatal thromboembolic complications [4], genital trauma [5], and fat embolism [6] to name a few.

Utmost caution in analysis of findings and 
formulating the medico legal conclusions should be practiced when such rare sequelae are encountered, because the categorization of hurt which plays a major role on the punishment and/or compensation of the offence during the medico-legal examination can be challenged by the court of law. Therefore, a detailed history and examination, considering the possible differential diagnosis, making relevant referrals in order to solicit specialist opinion becomes vital.

This paper discusses such rare complication of an interpersonal violence - rhabdomyolyis associated with Acute Kidney Injury.

\section{Case}

A 37-year-old man was brought to the hospital by a known person, who claims that this man was assaulted by a group of people, few hours prior to the admission. His past medical and surgical history was not known as the person who brought in the patient was not aware of it.

On admission, the patient was drowsy with the Glasgow Comas Scale (GCS) being 12/15, with eyes opening to pain (4), inappropriate responses through discernible words (3) and motor response to painful stimulus (5). Blood pressure was $90 / 60 \mathrm{mmHg}$ and heart rate was 88 beats/min. Physical examination showed multiple scalp haemotomas, focal contusions over the trunk and limbs and extensive grazed abrasions on the back of the chest. A fracture of the left leg and foot was suspected as there was gross swelling noted over the areas. His breath smelled of alcohol at the time of admission. Both lung fields were clear with equal air entry.

His non-contrast CT Brain and FAST scan (Focused Assessment with Sonography in Trauma) were unremarkable, and thereby injuries to brain and visceral organs were excluded. Fracture of the leg and foot were also excluded after relevant radiological investigations. Laboratory studies showed serum creatinine of $88 \mu \mathrm{mol} / \mathrm{L}$ rising up to $446 \mu \mathrm{mol} / \mathrm{L}$ within 24 hours of admission (normal being 70-115 $\mu \mathrm{mol} / \mathrm{L}$ ), serum urea $100 \mathrm{mg} / \mathrm{dL}$ (normal range $8-50 \mathrm{mg} / \mathrm{dL}$ ), blood Urea Nitrogen $42.43 \mathrm{mg} / \mathrm{dL}$, C-reactive protein $208.45 \mathrm{mg} / \mathrm{L}$, transaminase (aspartate aminotransferase, 4,393 U/L; and alanine aminotransferase, 2,491 U/L), along with marked elevation of serum creatinine phosphokinase (CPK) level $4984 \mathrm{U} / \mathrm{L}$ (normal range being 38-145 U/L). Serum potassium level was $6.3 \mathrm{mmol} / \mathrm{L}$ and sodium level was $133 \mathrm{mmol} / \mathrm{L}$. Full blood count revealed a neutrophilic white cell count of 31,190 and haemoglobin level of $14.4 \mathrm{~g} / \mathrm{dL}$ which dropped to $7.8 \mathrm{~g} / \mathrm{dL}$ in the following three days. Complete Urine Analysis (CUA) contained haemoglobin 3+, 27 red blood cells per high power field and was positive for leukocyte esterase. His Arterial Blood Gas Analysis revealed metabolic acidosis. Blood picture shows many polychromatic cells and occasional fragmented cells, and was concluded that non-immune haemolysis or Disseminated Intravascular Coagulation cannot be excluded.

A diagnosis of rhabdomyolysis was made. Shortly after admission, the patient's condition worsened and he developed oliguric acute kidney injury (AKI), and a cycle of haemodialysis was initiated.

Further history revealed that the patient has neither comorbidities nor past history of rhabdomyolysis.

In the following days, his renal functions improved and hemodialysis was discontinued. He was discharged after 11 days of hospital stay.

\section{Discussion}

This 37-year-old male who was brought into the hospital following an assault by a group of people, presented with altered level of consciousness, in addition to being under the influence of alcohol. There were extensive grazed abrasions and contusions involving his trunk and limbs. His FAST scan and NCCT_Brain were negative for any internal organ injuries. He gradually developed oliguria and his serum creatinine levels increased by many folds within 24 hours of admission. His liver enzymes, CRP and serum potassium levels were elevated, with a more than 35-fold increase of CPK. His white cell count was high and was predominantly neutrophillic and his urine contained red blood cells. His Arterial Blood Gas analysis revealed a metabolic acidosis.

Rhabdomyolysis is a common cause for oliguric renal failure, and can be traumatic [7] or non-traumatic [8] in origin, such as vigorous physical workouts, seizures, insect bites, drugs and toxins like alcohol, opioids and statins, infections, certain autoimmune diseases, endocrinopathies like hyper- or hypothyroidism, muscular dystrophies, metabolic disorders, hypokalaemia and septicaemia [9].

Rhabdomyolysis has specific clinical and laboratory parameters, but still requires high level of suspicion, so that the diagnosis is not missed. Patients with rhabdomyolysis may present with obvious muscle injuries or in the absence of injuries with muscle tenderness, localized or diffused pain, weakness and asymptomatic features like fatigue, nausea, vomiting, increased temperature, tachycardia and red or brown coloured urine [10]. Highly elevated levels creatinine phosphokinase (CPK), with values from 5 to 10 times the upper limit of normal is the most specific laboratory parameter for the diagnosis of rhabdomyolysis. Presence of myoglobinuria which is usually confirmed by the presence of red cells is also a reliable marker for the diagnosis of rhabdomyolysis. Elevated levels of lactase dehydrogenase and transaminase levels are also considered valuable markers of rhabdomyolysis [11]. But studies have shown the creatinine phosphokinase is more specific than other markers in diagnosing rhabdomyolysis [12]. Among the 
radiological investigations $\mathrm{MRI}$ is said to be superior to ultrasonography or CT in identifying affected muscles [13].

Acute Kidney Injury (AKI) is the most common recognized systemic complication of rhabdomyolysis. AKI is said to occur in 10 to $55 \%$ of cases with rhabdomyolysis, and is found to have a fatal outcome when there is coexisting multi-organ failure [14]. Arrhythmias, Acidosis, Compartment syndrome, Disseminated Intravascular Coagulation (DIC) and volume depletion a found to be other possible complications of rhabdomyolysis [15].

This case emphasizes the importance of bearing in mind the remote possibility of rhabdomyolysis in a patient with unexplained renal compromise and altered levels of metabolites. In clinical set-up, frequently Acute Kidney Injury following a trauma is thought to be a result of shock and the presence of dark urine is considered to be due to haematuria caused by bladder injuries. In this patient the highly elevated CPK, which is almost 35 times higher than the upper limit of normal is the most specific parameter to diagnose rhabdomyolysis, in association with elevated transaminase levels. Lactase dehydrogenase and urine myoglobin levels were not evaluated. But positive red cells and haemoglobin in the urine can be considered an indicator of myoglobinuria. Taking into account the clinical picture of the patient, his leg and foot swelling, could be sign of soft tissue oedema as a result of rhabdomyolysis and acute kidney injury. The rapid rise of serum creatinine levels in this case, is valid evidence of acute onset of diminishing renal functions. In the meantime, patient denies similar symptoms or symptoms indicative of kidney disease prior to the assault.

Rhabdomyolysis commences when the muscle contents are released into the circulation following an acute muscle injury [16]. These cellular contents from the damaged muscles lead to increased anion gap metabolic acidosis, hyperkalaemia, hyperphosphotaemia and hyperuriceamia [9] - two of these are observable in this case. Hypocalcaemia is thought to occur in cases of rhabdomyolysis, as the calcium is known to get deposited in damaged muscles, thereby causing a reduction in serum levels.

The main cause of AKI with a high mortality is rhabdomyolysis [17] with an incidence of $10-40 \%$. In this case the rapid rise of serum creatinine levels from $88 \mu \mathrm{mol} / \mathrm{L}$ rising up to $446 \mu \mathrm{mol} / \mathrm{L}$ within a day is due to the release of muscle constituents into the blood stream and reduced clearance due to diminishing renal functions [18]. Any compromised blood is compensated by an intravascular fluid shift, which usually takes at least 24-72 hours after the initial insult [19]. This explains the delayed drop in haemoglobin levels in this case. The very high neutrophilic counts are likely a result of the response to muscle injury and inflammation [20].
Various mechanisms have been explained to be causing $A K I$ due to rhabdomyolysis and they are renal vasoconstriction, formation of intratubular casts, direct toxic effects by myoglobin, renal ischaemia secondary to muscular vasoconstrictors, injury by the free radicals and disseminated intravascular coagulopathy $[21,22]$. Excessive leakage of extracellular fluid into injured muscle cells prompted by Renin-AngiotensinAldosterone system results in renal vasoconstriction [23]. Casts are formed when myoglobin is filtered through the glomerular basement membrane. During water absorption the concentration of myoglobin rises, and in the presence of acidic urine they accumulate and form obstructive casts. Free iron is formed when myoglobin fractions haeme ions, which in turn catalyze to form free radicals that are nephrotoxic. Substrates released from damaged muscle cells activate the coagulation cascade that can further enhance tubular obstruction. Interestingly, myoglobin is known to be less nephrotoxic in the absence of hypovolaemia and acidic urine.

To establish the diagnosis of rhabdomyolysis as the sole cause of AKI in this case with a vague history, it is important that the managing team excludes other possible causes such as pre-existing renal pathology, history of substance and alcohol abuse, usage of medications like statins and comorbidities such as seizures and endocrinopathies.

Once the managing medical team establishes the diagnosis of AKI due to rhabdomyolysis, it becomes an essential responsibility of the forensic pathologist to determine if the muscle injuries are a result of intentional trauma. In this case, an accidental fall, like one following a seizure can be excluded as the injuries are not confined to the anatomical prominences of the body. In the same manner, self-infliction of these injuries can be ruled out, as there are multiple injuries on the inaccessible sites of the body, such as the back aspect of the chest. Therefore, the causative agent of these injuries can be determined to be those caused by intentional violence.

Categorization of injuries in the medico-legal examination is of paramount importance in any case. But it is crucial in cases like this, not only because the diagnosis is principally made by the treating physician but also because these are very rare instances encountered by most of us, especially by the judicial system. If this patient was not attended to on time and if he had not been managed extensively to the level of subjecting him to haemodialysis he would have succumbed to his injuries. In other words, these injuries would definitely result in death in the absence of prompt and proper medical care. Therefore, the injuries were 'fatal in the ordinary course of nature' [24].

\section{Conclusion}

Rhabdomyolysis requires high index of suspicion 
when acute kidney injury and altered metabolite levels are suspected in a patient with major or minor muscle injuries. Therefore, forensic experts should always bear in mind the possibility of rhabdomyolysis and its fatal complications, when handling a case of physical violence, consisting of muscle trauma.

\section{Compliance with Ethical Standards and Consent}

This case report is about a clinical case scenario of a victim of physical abuse, who was eventually diagnosed with rhabdomyolysis associated with acute kidney injury. The examination was carried with informed written consent of the patient and his anonymity is maintained throughout the manuscript. This manuscript has not been submitted to any other journals, and is the original work of the authors and has not been published elsewhere.

\section{Conflict of Interest}

The authors declare that they have no conflict of interest.

\section{Adherence to National and International Regulations}

Not applicable.

\section{Consent for Publication}

Not applicable.

\section{Availability of Data and Material}

No objections in sharing data.

\section{Competing Interests}

The authors declare that they have no competing interests.

\section{Funding}

No funds used.

\section{References}

1. Cunningham RM, Carter PM, Ranney M, Zimmerman MA, Blow FC, et al. (2015) Violent reinjury and mortality among youth seeking emergency department care for assaultrelated injury: A 2-year prospective cohort study. JAMA pediatrics 169: 63-70.

2. Soa H-F, Nabib H (2018) Handlebar hernia-A rare complication from blunt trauma. Int J Surg Case Rep 49: 118-120.

3. Ulusoy E, Serpen B, Akgül F, Çitlenbik H, Sönmez DY, et al. (2018) Unexpected complication of blunt trauma: Evisceration. J Pediatr Emerg Intensive Care Med 5: 129131.

4. Andrew D (2009) Fatal thromboembolic complications following assault in a subject with an abdominal aortic aneurysm. Forensic Sci Med Pathol 5: 90-94.

5. Wright JL, Wessells H (2007) Urinary and Genital Trauma. In: WB Saunders, Penn Clinical Manual of Urology. 283309.
6. Cristina P, Mihaela S, Bogdan B, Adrian J, AndreeaAlexandra V, et al. (2019) Death due to a rare posttraumatic complication: Fat embolism. Journal of Military Medicine 122: $56-61$.

7. Bywaters EG, Beall D (1941) Crush injuries with impairment of renal function. Br Med J 1: 427-432.

8. Grossman RA, Hamilton RW, Morse BM, Penn AS, Goldberg M (1974) Nontraumatic rhabdomyolysis and acute renal failure. N Engl J Med 291: 807-811.

9. Esposito P, Estienne L, Serpieri N, Ronchi D, Comi GP, et al. (2018) Rhabdomyolysis-associated acute kidney injury. Am J Kidney Dis 71: A12-A14.

10. Torres PA, Helmstetter JA, Kaye AM, Kaye AD (2015) Rhabdomyolysis: Pathogenesis, diagnosis, and treatment. Ochsner Journal 15: 58-69.

11. Giannoglou GD, Chatzizisis YS, Misirli G (2007) The syndrome of rhabdomyolysis: Pathophysiology and diagnosis. Eur J Intern Med 18: 90-100.

12. Zimmerman JL, Shen MC (2013) Rhabdomyolysis. Chest 144: 1058-1065.

13. Moratalla MB, Braun P, Fornas GM (2008) Importance of MRI in the diagnosis and treatment of rhabdomyolysis. Eur J Radiol 65: 311-315.

14. Bosch X, Poch E, Grau JM (2009) Rhabdomyolysis and acute kidney injury. N Engl J Med 361: 62-72.

15. Keltz E, Khan FY, Mann G (2013) Rhabdomyolysis. The role of diagnostic and prognostic factors. Muscles Ligaments Tendons J 3: 303-312.

16. Aguiar DT, Monteiro C, Coutinho P (2015) Recurrent rhabdomyolysis secondary to hyponatremia in a patient with primary psychogenic polydipsia. Rev Bras Ter Intensiva 27: 77-81.

17. Zager RA (1989) Studies of mechanisms and protective maneuvers in myoglobinuric acute renal injury. Lab Invest 60: 619-629.

18. Harper J (1990) Rhabdomyolysis and myoglobinuric renal failure. Crit Care Nurse 10: 32-36.

19. Holcomb SS (2008) Third-spacing: When body fluid shifts. Nursing 38: 50-53.

20. Toumi H, F'guyer S, Best TM (2006) The role of neutrophils in injury and repair following muscle stretch. J Anat 208: 459-470.

21. Rosa EC, Liberatori Filho AW, Schor N, Lopes AC (1996) Rhabdomyolysis and acute kidney failure. Rev Assoc Med Bras 42: 39-45.

22. Holt SG, Moore KP (2001) Pathogenesis and treatment of renal dysfunction in rhabdomyolysis. Intensive Care Med 27: 803-811.

23. Petejova N, Martinek A (2014) Acute kidney injury due to rhabdomyolysis and renal replacement therapy: A critical review. Critical Care 18: 224.

24. Penal code of Sri Lanka. 